\title{
Ultra-Wideband Underwater Backscatter via Piezoelectric Metamaterials
}

\author{
Reza Ghaffarivardavagh*, Sayed Saad Afzal*, Osvy Rodriguez*, and Fadel Adib \\ Massachusetts Institute of Technology \\ ${ }^{*}$ Co-primary Authors \\ $\{$ rezagh,afzals, osvyrd,fadel $\} @$ mit.edu
}

\begin{abstract}
We present the design, implementation, and evaluation of $U^{2} B$, a technology that enables ultra-wideband backscatter in underwater environments. At the core of $U^{2} B$ 's design is a novel metamaterialinspired transducer for underwater backscatter, and algorithms that enable self-interference cancellation and FDMA-based medium access control.

We fabricated $U^{2} B$ nodes and tested them in a river across different weather conditions, including snow and rain. Our empirical evaluation demonstrates that $U^{2} B$ can achieve throughputs up to $20 \mathrm{kbps}$, an operational range up to $62 \mathrm{~m}$, and can scale to networks with more than 10 nodes. In comparison to the state-of-the-art system for underwater backscatter, our design achieves $5 \times$ more throughput and $6 \times$ more communication range. Moreover, our evaluation represents the first experimental validation of underwater backscatter in the wild.
\end{abstract}

\section{CCS CONCEPTS}

- Networks $\rightarrow$ Network architectures; • Hardware $\rightarrow$ Wireless integrated network sensors; • Applied computing $\rightarrow$ Environmental sciences;

\section{KEYWORDS}

Subsea IoT, Piezoelectricity, Backscatter Communication, Wireless, Energy Harvesting, Battery-free, Metamaterials, UWB

\section{ACM Reference Format:}

Reza Ghaffarivardavagh, Sayed Saad Afzal, Osvy Rodriguez, and Fadel Adib. 2020. Ultra-Wideband Underwater Backscatter via Piezoelectric Metamaterials. In SIGCOMM '20: 2020 Conference of the ACM Special Interest Group on Data Communication, August 10-14, 2020. ACM, New York, NY, USA, 14 pages. https://doi.org/10.1145/3387514.3405898

\section{INTRODUCTION}

Energy-efficient $\mathrm{u}$ nderwater $\mathrm{n}$ etworking $\mathrm{h}$ as $\mathrm{r}$ ecently witnessed mounting interest from academia and industry due to emergent needs for its environmental, defense, and industrial applications [20, 22, 24, 36]. Concerns about the impact of climate change on ocean health have prompted oceanographers and climatologists to seek sensor networks that can be used to monitor ocean vital signs such as carbon

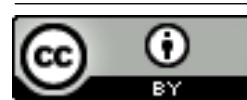

This work is licensed under a Creative Commons Attribution International 4.0 License. SIGCOMM '20, August 10-14, 2020, NY

(C) 2020 Copyright held by the owner/author(s).

ACM ISBN 978-1-4503-7955-7/20/08.

https://doi.org/10.1145/3387514.3405898 balance, coral reef conditions, and biodiversity [30, 32, 42]. On the defense front, DARPA launched the "Ocean of Things" program in 2017 to propel the development of low-cost, low-power distributed sensor networks that can enhance maritime situational awareness [8]. Major industry players - including Google, Microsoft, and Honeywell - have also become increasingly interested in deploying such networks to monitor underwater infrastructures ranging from oil and gas pipelines [14] to submerged datacenters [57].

Despite major advances in underwater technologies, existing proposals remain far from the vision of a low-cost, low-power distributed architecture for a subsea IoT. The majority of today's systems rely on point-to-point communication between modems that require at least 50-100 Watts for data transmission [33, 53], a power level that quickly drains the batteries of underwater sensors and limits their lifetime. Early workarounds for this high power consumption problem involved heavy duty-cycling which strangled the data rates to within few to tens of bits per second [21, 23]. Recent work on underwater wireless charging $[15,34]$ and backscatter [20] promises to extend battery life and to reduce the power consumption of underwater communication to sub-milliWatt levels; however, these systems are still limited in their throughput, operational range, and scale. For example, the state-of-the-art system for underwater backscatter is limited to $3 \mathrm{kbps}$ and a maximum distance of $10 \mathrm{~m} \mathrm{[20].}$

We present Ultra-wideband Underwater Backscatter $\left(U^{2} B\right)$, a system that enables scalable and ultra-low power underwater networking. The system brings the benefits of Ultra-Wideband (UWB) technology to low-power underwater communication. UWB is a mature technology in radio frequency (RF) communication ${ }^{1}$ and has been used to extend communication range [11], boost throughput [38], and scale radio networks via concurrent transmissions [18, 19]. However, existing UWB RF systems cannot be used for underwater communication because RF attenuates exponentially in water. This is why today's underwater communication systems primarily rely on acoustic signals, which have good propagation properties in water $[16,50]$.

Bringing UWB to underwater backscatter faces multiple challenges. First, underwater communication nodes, including backscatter nodes, rely on resonant piezoelectric transducers. These transducers have high efficiency when transmitting and receiving sound at their resonance frequency, but their performance quickly degrades as the frequency moves away from resonance. Hence, they are limited to narrow bandwidths. Second, backscatter communication is inherently full-duplex and suffers from self-interference between the transmitted signal and the backscatter response. This problem is exacerbated for UWB backscatter since UWB signals are typically below the noise floor making it more difficult to detect and

\footnotetext{
${ }^{1}$ A communication system is defined as UWB if its bandwidth is larger than $20 \%$ of its center frequency [51].
} 
decode them in the presence of strong self-interference. Finally, scaling underwater UWB backscatter to multiple nodes will introduce new forms of interference and require new mechanisms to disentangle interfering signals and decode them in frequency-selective and time-varying underwater environments.

At the heart of $U^{2} B$ 's approach to overcoming these challenges is a metamaterial-inspired transducer design for underwater backscatter. Metamaterials are artificial materials (or material composites) that exhibit properties which would otherwise not occur in nature. In the context of $U^{2} B$, we needed to develop a backscatter transducer that exhibits wideband properties.

Before we describe our new transducers, let us understand why traditional low-power designs have a limited bandwidth. Fig. 1(a) shows a typical underwater transducer consisting of a piezoceramic cylinder. Piezoceramic cylinders are used for transmitting and receiving sound underwater because they can transform sound to electric signals, and vice versa. The geometry of these transducers determines their resonance frequency. In the above figure, the resonance frequency is determined by the thickness, radius, and material composition of the piezoceramic cylinder. (By analogy, guitar strings have a resonance frequency that is determined by their thickness, length, and material). Existing underwater communication systems leverage this property to maximize the efficiency of backscatter and energy harvesting by transmitting sound at the resonance frequency of the transducer. ${ }^{2}$ However, the signal-to-noise ratio (SNR) and energy harvesting efficiency significantly degrade outside this frequency (as shown in Fig. 1(a)). In principle, it is possible to use a non-resonant transducer; however, such a design is undesirable as it would be inefficient and would further limit the range and SNR of underwater communication [10, 43].

To overcome the bandwidth limitation while maintaining high efficiency, $U^{2} B$ synthesizes different forms of resonance through the multi-layer (metamaterial) design shown in Fig. 1(b). The design alternates between active (piezoelectric) layers and passive (polymer) layers. It makes use of two kinds of resonance: the first (shown in blue and yellow) is similar to that of standard transducers and arises from the primary resonance of each individual piezoceramic layer. The second and more interesting, kind of resonance arises from the passive coupling between the different active layers. Even though the polymer layers are themselves passive, by sandwiching them between two piezoceramic layers, we impose new constraints on how the active layers may stretch and squeeze concurrently. This coupled interaction yields additional resonances that fill in the gaps between the first two types of resonances, thus resulting in wideband operation (depicted by green curves in Fig. 1(b)). Each of these resonances corresponds to a complex vibration mode - called eigenmode. The combination of these eigenmodes allows $U^{2} B$ to achieve high-efficiency backscatter over a wide bandwidth. In $\$ 3.1$, we explain this multi-layered design in detail, and in $\$ 4.1$, we describe how $U^{2} B$ can independently or jointly activate different layers for communication and energy harvesting.

A major benefit of UWB RF technologies is that they can extend the communication range between two nodes by allowing them to

\footnotetext{
${ }^{2}$ While past systems have tried to shift the resonance electrically [20], they can only do so by a small amount (around $2-3 \mathrm{kHz}$ ) but remain limited by the mechanical resonance of the piezoceramic cylinder's geometry.
}

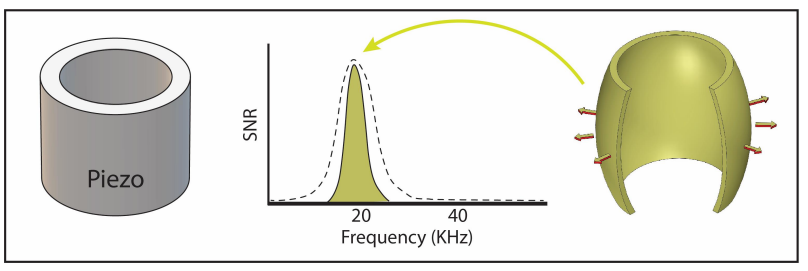

(a) Traditional Single-Layer Resonator

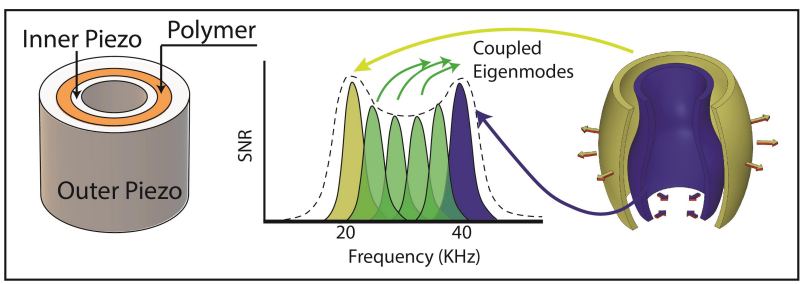

(b) $U^{2} B$ 's Metamaterial Design

Figure 1- $U^{2} B$ Synthesizes Resonances to Achieve UWB Performance. In each of (a) and (b), the left figure shows the transducer architecture, the middle figure shows the SNR as a function of frequency, and the right figure shows one of the vibration modes or eigenmodes - of the active layers.

decode packets that are received below the noise floor [45]. Specifically, a UWB transmitter can apply a spread-spectrum code (e.g., CDMA) before transmitting its packet, and a UWB receiver can correlate with the same code to boost the received signal and decode packets that are orders of magnitude below the noise floor. ${ }^{3}$ In the context of underwater backscatter, however, the communication range is not only limited by the noise floor, but also by the strong self-interference between the transmitted and received signals (since the nodes communicate by modulating the reflection of a continuous downlink signal sent from a remote transmitter). This self-interference limited past systems' operational range to within a few meters [20].

To overcome this challenge, $U^{2} B$ exploits the multi-resonant wideband transducer and shifts the backscatter response to an out-of-band channel. Specifically, $U^{2} B$ 's ultra-wide bandwidth allows it to divide its available spectrum to multiple orthogonal frequency channels and to use separate channels for downlink and uplink communication. Let us assume the downlink packet is transmitted at $40 \mathrm{kHz}$. The backscatter node can leverage the non-linear nature of backscatter to shift the uplink response to a different center frequency, e.g., $20 \mathrm{kHz}$. A remote receiver that obtains both the strong downlink (at $40 \mathrm{kHz}$ ) and weak backscatter uplink (at $20 \mathrm{kHz}$ ) can simply filter out the downlink signal in hardware. This approach allows it to cancel self-interference and decode the weak backscatter packet even if it is orders of magnitude weaker than the transmitted signal or the noise floor. In $\$ 3.2$, we describe this approach in detail and demonstrate how it can increase the communication range by an order of magnitudes over state-of-the-art designs.

$U^{2} B$ 's design builds on the above primitives to address many of the limitations of prior low-power subsea IoT proposals. Beyond increasing the communication range, it uses the wide bandwidth to boost throughput and increase the number of frequency channels

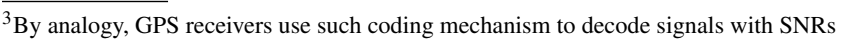
of $-20 \mathrm{dBm}$
} 
available for communication, thus scaling the subsea IoT to dozens of nodes.

We implemented a prototype of $U^{2} B$ and tested it in a river and indoor pools. We mechanically fabricated our metamaterial transducers in-house; our fabrication process (detailed in \$4) involved designing and 3D printing the molds, stacking (electrically and mechanically) piezoceramic transducers, and curing the transducers under high-pressure environments. Our prototype evaluation was performed with integrated energy-harvesting micro-controllers as well as with software radio-based nodes (USRP N210 [3] with LFRX daughterboards [2]). Our empirical evaluation across around 400 experimental trials demonstrated the following results:

- In comparison to single-layer backscatter nodes, $U^{2} B$ 's design boosts the throughput from $3 \mathrm{kbps}$ (of the state-of-the-art system) to more than $20 \mathrm{kbps}$. Such throughput is considered on the high end of underwater communication systems, and can enable new applications such as streaming low-resolution images (e.g., for coral reefs or aquafarms).

- $U^{2} B$ 's self-interference cancellation approach allows it to mitigate the direct signal from the projector. Practically, we show that it enables us to communicate at up to $60 \mathrm{~m}$.

- Our evaluation also demonstrates how $U^{2} B$ can support up to 10 concurrent transmissions (before spatial reuse), and that it can adapt its throughput based on the distance and size of the network.

Contributions: $U^{2} B$ is the first system that enables ultra-wideband underwater backscatter. Its design introduces a novel metamaterialbased transducer that synthesizes multiple resonances to achieve wideband operation. The design brings various capabilities to the subsea IoT domain, including out-of-band self-interference cancellation and multi-channel communication. The paper also contributes a prototype implementation and evaluation in challenging real-world environments.

\section{THE NARROWBAND PROBLEM}

We start by explaining why existing solutions for underwater backscatter have a limited bandwidth. Then, in $\$ 3$, we explain how $U^{2} B$ overcomes this problem by introducing an ultra-wideband design.

\subsection{Underwater Backscatter}

Before delving into the narrowband problem, we provide a short primer of underwater backscatter and refer the interested reader to [20] for a more detailed explanation. Fig. 2 shows a simplified example of an underwater backscatter system, consisting of a projector (transmitting speaker), hydrophone (receiver), and a backscatter node. The projector sends an acoustic signal which reflects off the backscatter node and returns to the hydrophone. ${ }^{4}$ The backscatter node communicates by modulating its reflections. Specifically, it can send bits of zero and one by alternating between reflective and nonreflective states. The hydrophone records the received signals and senses changes in reflection power in order to decode the transmitted bits.

\footnotetext{
${ }^{4}$ The signal also arrives on a direct path from the projector to the hydrophone, but we ignore this path for simplicity, and we revisit it in $\$ 3.2$.
}

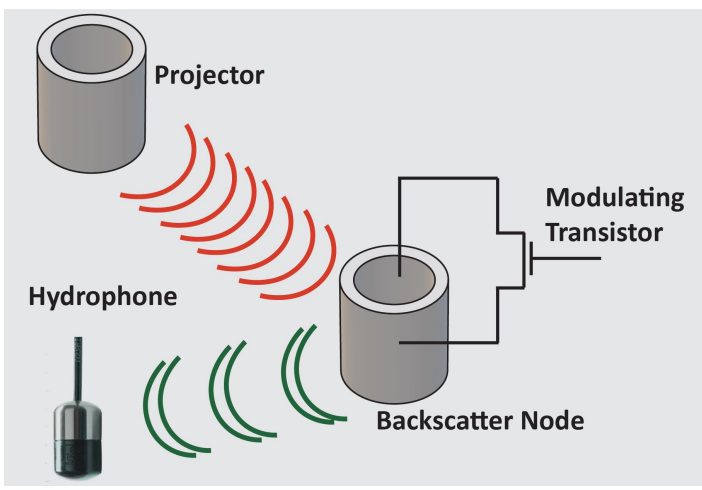

Figure 2-Underwater Backscatter. In underwater backscatter, a projector transmits an acoustic signal on the downlink, which reflects off the backscatter node and comes back to the hydrophone receiver. To communicate information, the node can change its reflection coefficient by controlling an impedance switch across the terminals of a piezoelectric front-end.

The signal-to-noise ratio (SNR) of the received signal is determined by the difference between the reflective and non-reflective states. It is given by the following equation:

$$
S N R=\frac{\left|P_{\text {reflective }}-P_{\text {non-reflective }}\right|^{2}}{|N|^{2}}
$$

where $P_{\text {reflective }}$ and $P_{\text {non-reflective }}$ refer to the pressure received by the hydrophone ${ }^{5}$ in the reflective and non-reflective states and $N$ refers to the amplitude of noise. The square indicates the power.

We can simplify the above equation by rewriting it as a function of the reflection coefficient $\Gamma$ and the incident pressure $P_{\text {incident }}$ as follows:

$$
S N R=\left|\Gamma_{\text {reflective }}-\Gamma_{\text {non-reflective }}\right|^{2} \frac{\left|P_{\text {incident }}\right|^{2}}{|N|^{2}}
$$

To modulate the reflection coefficient of sound, underwater backscatter nodes rely on piezoelectric materials. Specifically, by opening and shorting the terminals of that material (via a switch as shown in Fig. 2), they can toggle between the two reflective states, thus enabling backscatter communication.

\subsection{The Resonance Bottleneck}

While using piezoelectric materials enables underwater backscatter, it limits the bandwidth of underwater communication. Specifically, piezoelectric materials must be operated at a specific resonance frequency - also called the natural frequency of the material. At resonance, they vibrate with a large amplitude, which leads to high efficiency in sending and receiving sound. A popular example of resonance is how an opera singer can break glass with her voice $[40,41]$; in particular, when the singer's voice matches the resonance frequency of the glass, the glass vibrates with higher and higher amplitudes and eventually breaks [40, 41]. Similarly, when piezoelectric materials are excited by their resonance frequency, they generate stronger signals.

To gain more insight into the bandwidth problem, we simulated a piezoceramic cylinder's vibration at different frequencies and measured the amplitude of vibration. Fig. 3 visualizes the amplitude

\footnotetext{
${ }^{5}$ Note that the amplitude of the electric field in RF is replaced by the amplitude of the pressure wave in acoustics.
} 

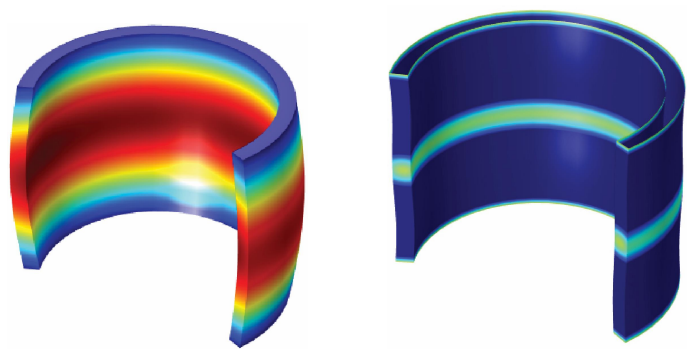

(a) At $f=f_{\text {resonance }}$

(b) At $f \neq f_{\text {resonance }}$

Figure 3-Single-Layer Eigenmode. The figure shows the amplitude of the piezo vibration across its body when it is excited (a) at resonance, and (b) outside resonance. The vibrations are visualized as a heat map where red and blue represent high and low vibration amplitudes respectively.

as a heatmap over the cross-section of the piezoelectric cylinder. ${ }^{6}$ The heatmap shows the largest amplitude of vibration in red and the lowest amplitude of vibration in blue. Fig. 3(a) shows that when the cylinder is excited by its resonance frequency, it expands and shrinks in the radial direction with a large amplitude; this radial vibration is called an eigenmode and the corresponding frequency is called an eigenfrequency. Fig. 3(b) shows that when the same cylinder is excited by a frequency far from its resonance, the vibration amplitude is very small. This simulation verifies that operating at resonance is necessary to achieve high efficiency in transmitting and receiving sound. What is less clear - and has not been well-studied in past work - is why resonance limits the underwater backscatter bandwidth of piezoelectric materials. The rest of this section focuses on understanding this problem.

The impact of resonance on SNR. Recall from Eq. 1 that the SNR depends on the difference between the reflection coefficients in the two backscatter states. The reflection coefficient itself is a function of the acoustic impedances of water and the piezoelectric material. Mathematically, the reflection coefficient can be described using the following equation:

$$
\Gamma=\frac{Z_{\text {piezo }}-Z_{\text {water }}}{Z_{\text {piezo }}+Z_{\text {water }}}
$$

where $Z_{\text {piezo }}$ and $Z_{\text {water }}$ refer to the acoustic impedances of the piezoceramic cylinder and water. During backscatter, the terminals of the piezoelectric are opened and shorted, resulting in different piezoelectric impedances $Z_{\text {piezo }}$ across the two states. This, in turn, modulates the reflection coefficient and enables backscatter.

Next, we would like to understand the impact of frequency on the SNR. Fig. 4 plots the absolute value of the difference in reflection coefficients (between open and short cases) as a function of frequency and as a function of the impedance of $Z_{\text {piezo. }}$. We make the following remarks from the figure:

- At the resonance frequency, the difference in reflection coefficients between the two states is maximized, resulting in the highest SNR. The SNR degrades quickly outside resonance.

- The figure also plots the difference in reflection coefficients as a function of the imaginary part of the impedance. It demonstrates that the SNR is maximized when the imaginary component of the

${ }^{6}$ We used the COMSOL Multiphysics software [1] to simulate the vibrations of the piezoceramic cylinder.

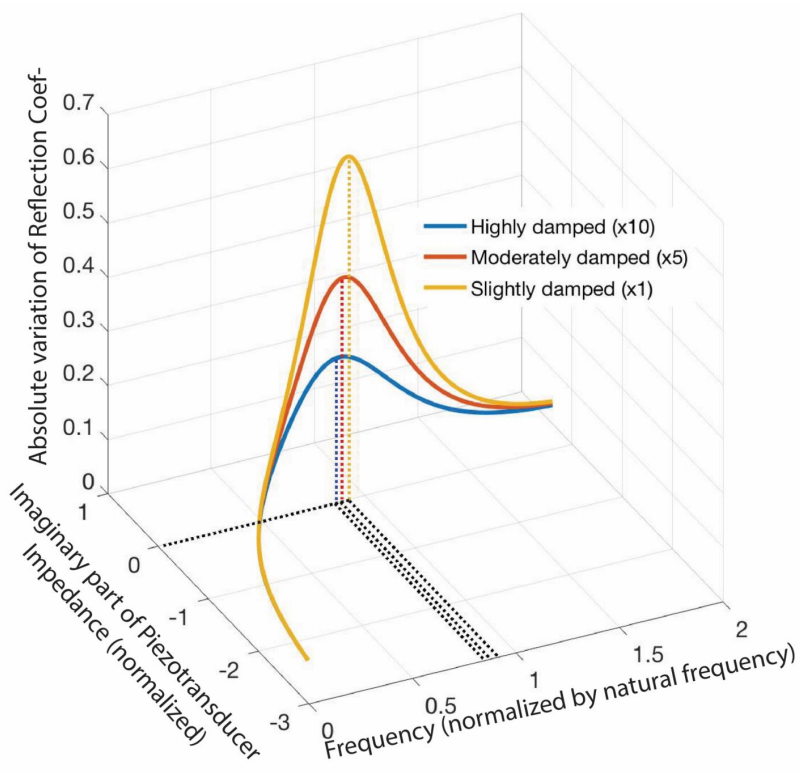

Figure 4-Backscatter and Resonance. The figure plots the absolute value of the difference in the reflection coefficient (between open and short cases) as a function of normalized frequency and as a function of the normalized imaginary component of piezo-transducer impedance.

impedance is zero. Intuitively, the imaginary part is zero when the vibration of the cylinder's wall and the acoustic wave are in-phase (i.e, the phase $\phi$ of the complex impedance is zero). In reference to the opera singer analogy, when the singer's voice and the glass vibrate in-phase, the glass's amplitude increases.

We can also show this mathematically. The impedance is a function of the cylinder's mass $M$, its stiffness $K$, and its resistance $R$. Formally, we show in the appendix that the backscatter acoustic impedance can be given by the following equation:

$$
Z_{\text {piezo }}=R+j \omega\left(M-\frac{K}{\omega^{2}}\right)
$$

The above equation reaffirms that the absolute value of the impedance is minimized when its imaginary part is zero. The equation also shows that the resonance frequency is $\omega=\sqrt{K / M}$. - The figure repeats the same simulation for different resistance values $R$, which correspond to different damping coefficients. While more damping leads to lower SNR, it does not impact the resonance frequency.

Before we move on to describe $U^{2} B$ 's approach to overcoming this narrowband problem, it is worth reflecting on the difference in effective bandwidth between underwater piezoelectric backscatter and RF backscatter (e.g., RFIDs). RF backscatter operates at a much higher center frequency $(\mathrm{GHz})$ than acoustic/ultrasonic backscatter $(\sim$ tens of $\mathrm{kHz}$ ). As a result, a communication bandwidth of $20 \mathrm{kHz}$ for piezoacoustic backscatter would be of the same order as its center frequency, but that same bandwidth would be less than $1 \%$ the center frequency of RF communication. As per Fig. 4, this makes it significantly more difficult for piezo-acoustic backscatter than for RF backscatter to achieve high SNR across the same bandwidth (since the absolute value of the difference in reflection coefficient is a function of the normalized frequency). 

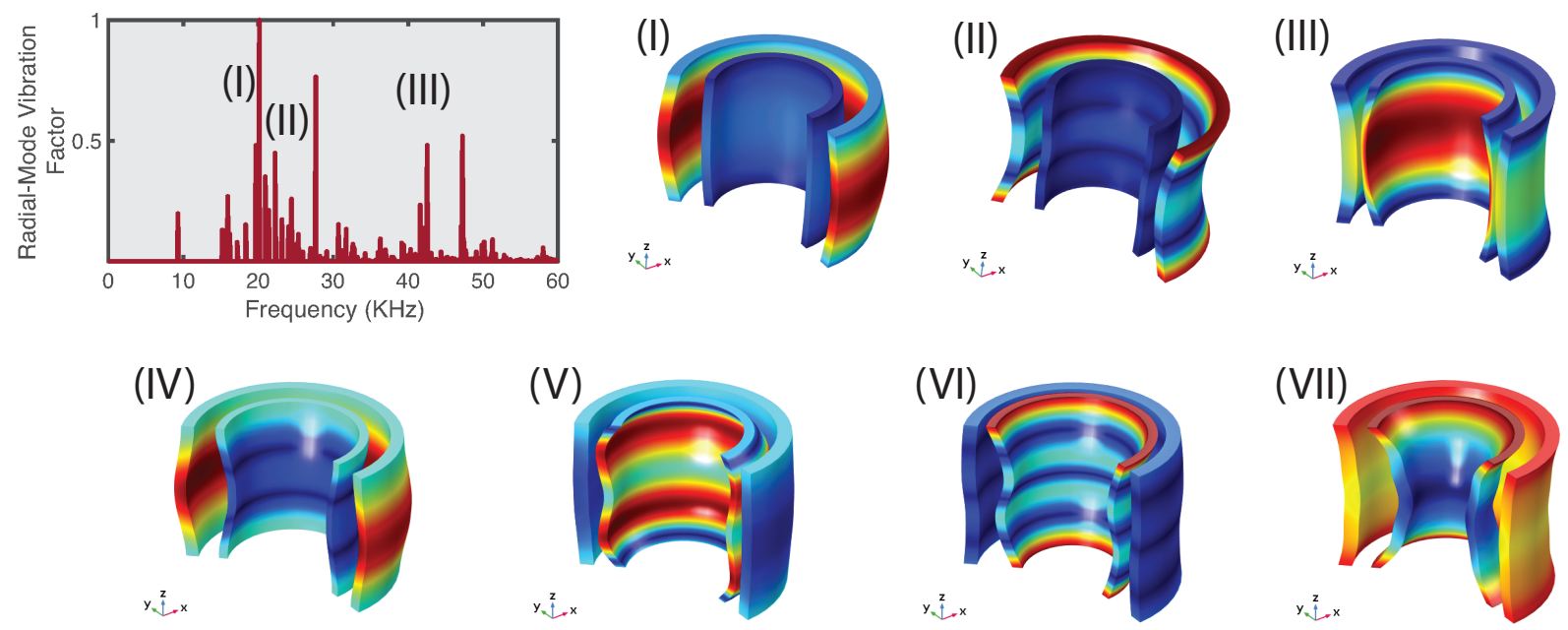

Figure 5-Eigenmodes and Eigenfrequencies of $U^{2} B$ 's Metamaterial Design. The figure in the top left shows the vibration amplitude across the different eigenfrequencies. (I)-(VII) depict the eigenmodes associated with some of the eigenfrequencies. The vibration amplitude for each of these eigenmodes is visualized as a heatmap across the body of the transducer, where red indicates a high amplitude vibration and navy blue indicates low amplitude vibration.

\section{ULTRA-WIDEBAND META-MATERIAL DESIGN}

So far, we have explained why existing solutions for underwater backscatter suffer from a narrow communication bandwidth. In this section, we describe how $U^{2} B$ overcomes this challenge via a metamaterial design, and how its design boosts the throughput, range, and scale of underwater backscatter networking.

\subsection{Coupled Eigenmodes}

A strawman solution to the bandwidth problem is to connect multiple piezoelectric cylinders, each having a different resonance frequency. However, such an approach would result in bulky and costly nodes, and it does not scale well to a wide bandwidth since it requires adding a new cylinder for each frequency. The design would also suffer from a directionality problem since placing multiple cylinders side-byside would mimic a fixed antenna array (beamforming) behavior.

To provide a scalable, cost-effective, compact, and omnidirectional solution, $U^{2} B$ adopts a multi-layer architecture that alternates between active and passive layers. This architecture is shown in Fig. 1(b) and consists of two active piezoelectric layers and a passive polymer layer sandwiched between them.

Despite its apparent simplicity, $U^{2} B$ 's multi-layer design has powerful properties that enable it to operate over a wide bandwidth. First, because its layers are concentric, the transducer does not suffer from a directionality problem. Second, because the polymer between the two active layers is mechanically compliant (i.e., flexible), it allows the inner and outer layers to vibrate independently to some extent. This enables the overall structure to inherit the Eigenfrequency and Eigenmode of both Piezoelectric layers without suffering from the problems of the strawman approach described earlier. Finally, even though the polymer itself is flexible, it imposes new constraints on how the two layers can vibrate with respect to each other, thus resulting in a coupled behavior. This coupling provides additional degrees of freedom that lead to new Eigenmodes and Eigenfrequencies that would not be present in any of the individual active piezoelectric layers.

We simulated $U^{2} B$ 's design at different frequencies and show some of the resulting Eigenmodes and Eigenfrequencies in Fig. 5. Similar to our earlier visualization, we overlay a heatmap of the vibration amplitude on the cross-section of each of the two layers. Consider mode (I) in the center top of the figure. This mode results from exciting the transducer at a frequency of $20 \mathrm{kHz}$, and it corresponds to the original radial vibration of the outer piezoelectric cylinder. Notice how, in this configuration, the outer layer exhibits large vibrations, while the inner layer is relatively static. This verifies that $U^{2} B$ can indeed inherent the eigenmode of its active layers.

Next, consider mode (III) in the top right of the figure. This mode is interesting because both the inner and outer piezos exhibit large radial vibrations as demonstrated by the red and yellow central regions in both of them. This eigenmode corresponds to the scenario where both layers vibrate by bending in opposite directions to each other. Physically, this mode arises from the fact that both piezoelectric cylinders have the same height, which results in a bending mode vibration that is enhanced by stacking them together. ${ }^{7}$

It is important to note that not all eigenmodes of this multi-layer design have a simple physical interpretation. In particular, the bottom row of Fig. 5 shows a number of Eigenmodes which would not have been possible without the coupling between the two active layers. In mode (V), for example, we can see that the internal layer itself splits into two concurrent radial modes in the top and bottom half of the cylinder. Such a vibration mode would not be natural for a single-layer piezoelectric cylinder in the absence of coupling.

The top left of Fig. 5 plots the vibration amplitude as a function of frequency. Surprisingly, the figure demonstrates that $U^{2} B$ has a large number of eigenmodes between $20 \mathrm{kHz}$ and $60 \mathrm{kHz}$ due to its coupled multi-layer design. Mathematically, it is possible to derive these eigenmodes by solving the following differential

\footnotetext{
${ }^{7}$ Note that this bending mode would also exhibit for each cylinder alone, but would be weaker than the radial mode (I).
} 




Figure 6-Characteristic Curve for Single and Multi-layer Designs. The figure plots the characteristic curve for single-layer (in red) and multi-layer (in black) designs as a function of frequency.

equation, along the different degrees of freedom of this meta-material structure [31]:

$$
\operatorname{det}\left(\omega^{2} M-K\right)=0
$$

To solve this differential equation, we used the COMSOL Multiphysics software [1], and we defined the coupling between the layers (both active and passive) by applying continuity constraints on both pressure and velocity across the boundaries. The wealth of resonances arising from this multi-layer design signals that $U^{2} B$ would indeed exhibit the desired ultra-wideband behavior. Specifically, the presence of multiple Eigenmodes and Eigenfrequencies would ensure that the backscatter SNR remains sufficiently high over a wide bandwidth.

Experimental Validation in a River. Next, we would like to empirically verify that $U^{2} B$ exhibits wideband behavior. We ran an experiment to compare the SNR of a $U^{2} B$ node to that of a state-ofthe-art underwater backscatter node, PAB, which uses a single-layer piezoelectric resonator. Our experiments were performed in the river which had a depth of around $4 \mathrm{~m}$. In these experiments, the acoustic transmitter sends a downlink signal at a frequency of $37.5 \mathrm{kHz}$. The backscatter node modulates this signal at different rates, and the hydrophone measures the SNR of the received signal at the corresponding frequency. (The design of the backscatter node is detailed in $\$ 4)$.

Fig. 6 plots the SNR of the received signal as a function of frequency for both single-layer (PAB, in red) and multi-layer $\left(U^{2} B\right.$, in black) designs. The figure plots the exact values of the measured SNR with dotted-dashed lines, and the smoothed SNR curves with solid lines. In particular, since the experiment is performed in a river (i.e., an uncontrolled testing environment), the communication channel is impacted by various factors - including wind, river current, and depth - and changes rapidly over time. These changes in the channel impact the result by creating high-frequency variations that can be seen in the exact values of the resulted SNR. Therefore, we rely on the smoothed/averaged version of the SNR, shown with the solid line, to characterize the performance of the two designs.

We make the following observations:

- First, the overall SNR of a $U^{2} B$ node is higher than that of the single-layer node, $\mathrm{PAB}$, across all frequencies. The increased SNR results in higher channel capacity - which enables achieving higher throughputs (as we demonstrate empirically in \$5.1) - and enables communication over further distances (as we demonstrate empirically in 55.2 ).
- Second, in the single-layer PAB design, the backscatter SNR is relatively limited to around $20 \mathrm{kHz}$ (from $10-30 \mathrm{kHz}$ ); outside this band, the SNR significantly decays due to being far from resonance. The limited bandwidth in PAB's design limits both the throughput and the available spectrum for communication. In contrast, $U^{2} B$ 's multi-layered design exhibits stronger SNR performance over a wide bandwidth due to its coupled resonance modes. Specifically, the backscatter signal's SNR in $U^{2} B$ remains sufficiently high across the entire band spanning $10 \mathrm{kHz}$ to $60 \mathrm{kHz}$.

- Interestingly, $U^{2} B$ achieves higher SNR than PAB even between $10 \mathrm{kHz}$ and $30 \mathrm{kHz}$. There are two reasons for this improvement. First, the acoustic impedance matching for $U^{2} B$ is higher than that of PAB; this is because the added polymer layer in $U^{2} B$ reduces its density, making its acoustic impedance closer to that of water. Second, even though the downlink frequency in this region is close to only one of the active piezoceramic layers, the other layer would still vibrate, thus enhancing the overall efficiency of $U^{2} B$.

- It is worth noting that the backscatter performance is not frequency flat and exhibits a dip around $35 \mathrm{kHz}$. This behavior mimics the theoretical simulation in the top left of Fig. 5, which also exhibits a dip around that frequency.

- Another point worth noting is that the single-layer node exhibits a small peak around $55 \mathrm{kHz}$. This peak is corresponds to the bending eigenmode due to the length (rather than radius) of the cylinder. This corresponds to mode (III) in Fig. 5, and is reinforced in the multi-layer structure due to coupling.

\subsection{Self-Interference Cancellation}

Backscatter communication is inherently full duplex, since the backscatter node communicates by modulating the reflections of a downlink signal. As a result, the hydrophone receives a strong downlink signal from the projector as well as a weak backscatter reflection from a low-power node. Unfortunately, the projector's direct signal can overwhelm the receiving hydrophone and prevent it from detecting the weak backscatter response. Note that increasing the transmit power from the projector would not solve the problem because it boosts both the downlink signal as well as the backscatter response. In fact, boosting the transmit power would saturate the analog-to-digital converter (ADC) of the receiver and prevent it from being able to sense the backscatter response even if the backscattering node is nearby.

To better understand this challenge, let us assume that the projector transmits the downlink signal at $f_{c}=40 \mathrm{kHz}$, which falls well within the bandwidth of $U^{2} B$ 's front-end as per Fig. 6. Additionally, let us assume that the backscatter node wishes to transmit a packet $p(t)$ on the uplink. To do so, the node can simply apply $p(t)$ across the backscatter switch, resulting in the following signal being received by the hydrophone:

$$
y(t)=I \cos \left(2 \pi f_{c} t\right)+h p(t) \cos \left(2 \pi f_{c} t\right)
$$

where $I$ is the amplitude of the direct path from the projector and $h$ is the channel from the backscatter node to the receiver. Because $I>>h$, we will have self-interference which limits the ability to sense the backscatter response of a faraway node.

To overcome this challenge, $U^{2} B$ leverages its wideband frontend, shifts the backscatter response out-of-band, and filters out the 


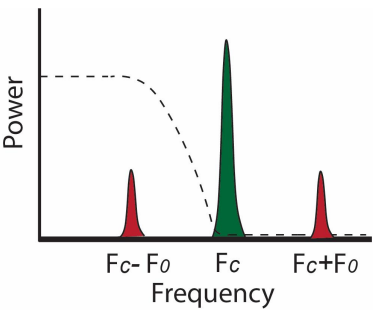

(a) Self-interference Cancellation

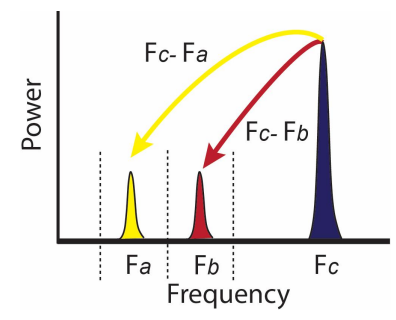

(b) FDMA MAC
Figure 7-Scaling the Range and the Size of $U^{2} B$ networks. The figure plots the power of the signal received by a hydrophone as a function of frequency. (a) shows how $U^{2} B$ can eliminate the downlink self-interference (in green) and retain a backscatter response (in red). (b) shows how $U^{2} B$ can scale to multiple nodes by assigning a different frequency channel to each of them.

strong in-band downlink signal as shown in Fig. 7(a). Specifically, rather than just applying $p(t)$ across the backscattering switch, it can apply $p(t) \cos \left(2 \pi f_{o} t\right)$ to shift the response away from the downlink signal in the frequency domain. In such a scenario, the received signal by the hydrophone is given by the following equation:

$$
\begin{aligned}
y(t)=I \cos \left(2 \pi f_{c} t\right) & +h p(t) \cos \left(2 \pi f_{c} t\right) \cos \left(2 \pi f_{o} t\right) \\
=I \cos \left(2 \pi f_{c} t\right) & +0.5 h p(t) \cos \left(2 \pi\left(f_{c}-f_{o}\right) t\right) \\
& +0.5 h p(t) \cos \left(2 \pi\left(f_{c}+f_{o}\right) t\right)
\end{aligned}
$$

So, the received signal contains the downlink frequency at $f_{c}$ and the backscatter response at $f_{c}-f_{o}$ and $f_{c}+f_{o}$. Assuming $f_{o}=25 \mathrm{kHz}$, then the backscatter response is at $15 \mathrm{kHz}$ and $65 \mathrm{kHz}$, while the downlink signal remains at $40 \mathrm{kHz}$ as shown in Fig. 7(a). Thus, the receiver can simply apply a low-pass filter whose cutoff is $30 \mathrm{kHz}$ to eliminate the downlink signal. This leaves it with only the backscatter response at $15 \mathrm{kHz}$ allowing it to easily decode it.

Few points are worth mentioning about $U^{2} B$ 's approach for selfinterference cancellation:

- First, by mitigating the strong downlink signal, the projector can transmit higher power and receive a stronger response from further away backscatter node. We can also place a low-noise amplifier (LNA) after the filter but before the ADC to further improve the sensitivity of our receiver to a further away node. Our implementation employs both of these techniques to significantly boost the range of communication of underwater backscatter as we empirically demonstrate in $\$ 5$.

- In our above discussion, we employed a low-pass filter to eliminate the downlink signal. Alternatively, it is possible to employ a high-pass filter (e.g., around $50 \mathrm{kHz}$ in the above example) to mitigate self-interference. In such an implementation, the receiver retains the backscatter response at $65 \mathrm{kHz}$ and can still decode it. Naturally, it can also employ a notch filter around $40 \mathrm{kHz}$ and retain both $15 \mathrm{kHz}$ and $65 \mathrm{kHz}$ to obtain an even better SNR.

- One might wonder whether it is possible to simply apply a notch filter without shifting the backscattered response out of band. While this is possible in theory, in practice, it would require a very high-Q filter which may be infeasible and it would result in mitigating not only the self-interference, but also the backscatter response [28].

- Another interesting question is whether applying $f_{o}=25 \mathrm{kHz}$ would defeat the purpose of backscatter since it is of the same order of the downlink frequency $f_{c}=40 \mathrm{kHz}$. Recall that the primary benefit of backscatter is that the receiver does not need to amplify its own generated signal since it communicates by reflecting a powerful downlink signal. In practice, we found that if the hydrophone is placed very close to a $U^{2} B$ node, it would indeed sense a much weaker signal at $f_{o}$ in comparison to a much stronger response at $f_{c}-f_{o}$ and $f_{c}+f_{o}$. This verifies the benefits of backscatter despite the apparent concern.

- It is worth noting that out-of-band backscatter has been explored in the context of RF communication [28, 56]; however, prior designs couldn't adapt it to underwater backscatter because they had a limited bandwidth [20]. By introducing an ultra-wideband metamaterial design, $U^{2} B$ can bring the benefits of out-of-band backscatter to underwater systems.

\subsection{Scaling to Many Nodes}

Next, we describe how $U^{2} B$ leverages its ultra-wideband performance to scale underwater backscatter networks to a larger number of nodes. At a high level, the system employs an FDMA style MAC protocol to enable concurrent transmissions. It divides the overall available bandwidth into different sub-channels and allocates each sub-channel to a different backscatter node. Because $U^{2} B$ enjoys a wider bandwidth in comparison to prior designs, it can support a larger number of concurrent transmissions with minimal interference. $^{8}$

To understand how $U^{2} B$ supports concurrent transmissions, let us consider a simple network with two backscatter nodes $A$ and $B$, and let us assume that the projector wants to allocate channels centered at $f_{A}$ and $f_{B}$ to these two nodes, as shown in Fig. 7(b). To do so, the projector needs to instruct each of the two nodes to shift by the appropriate frequency in order to occupy their respective channels. Hence, it sends a downlink command signal, instructing node $A$ to apply a shifting frequency of $f_{c}-f_{A}$ and node $B$ to apply a shifting frequency of $f_{c}-f_{B}$, which would result in the desired shifts as shown in the figure.

Mathematically, the hydrophone receives the following signal:

$$
\begin{aligned}
y(t)=\left(I+h_{A} S_{A}(t) \cos \left(2 \pi\left(f_{c}-f_{A}\right) t\right)+\right. \\
\left.\quad h_{B} S_{B}(t) \cos \left(2 \pi\left(f_{c}-f_{B}\right) t\right)\right) \cos \left(2 \pi f_{c} t\right)
\end{aligned}
$$

This results in a shifting behavior similar to that described in $\$ 3.2$, as demonstrated in the figure. Subsequently, the receiver can simply apply a bandpass filter around $f_{A}$ and $f_{B}$ to decode the two concurrent transmissions. The same idea can be extended to a large number of nodes, each occupying a different subchannel $f_{i}$.

Two additional points are worth noting:

- Due to the on-off switching nature of backscatter communication, the resulting backscatter signal is a square wave (rather than a simple cosine). One challenge, here, is that the harmonics of the square wave may interfere with other nodes by overlapping with their channels. To this end, the projector can pre-determine orthogonal channels (i.e., ones whose harmonics don't interfere) in its channel allocation process. Moreover, $U^{2} B$ adapts its MAC protocol to maximize the spacing between the allocated channels, thus minimizing interference.

\footnotetext{
${ }_{8}^{8}$ Note that PAB could support 2 concurrent transmissions, but required two hydrophones. In contrast, $U^{2} B$ can support a much larger number of backscatter nodes even with a single hydrophone receiver.
} 

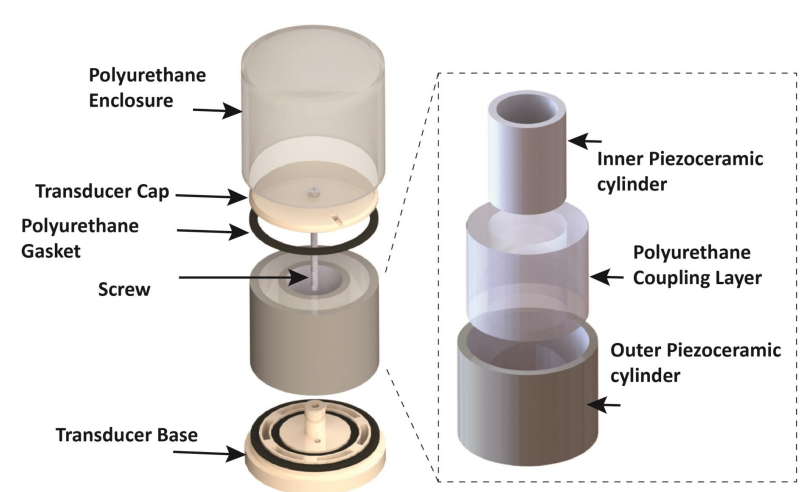

Figure 8-Exploded Transducer View. The figure shows the exploded transducer view and the layered piezoceramic structure for $U^{2} B$.

- When reasoning about the overall behavior of $U^{2} B$, it is important to distinguish between two different frequency/vibration concepts: The first is the metamaterial mechanical vibration; when the metamaterial is excited at a certain frequency, it automatically vibrates at that frequency's corresponding eigenmode (as shown in Fig. 5). The second concept is the backscatter frequency, which is determined by how the backscatter switch is modulated (e.g., by a micro-controller). To program the backscatter frequency, the transmitter sends a command signal on the downlink instructing the node to modulate the backscatter switch, resulting in an uplink frequency $f_{A}$. As a result, both the downlink frequency and the uplink frequency are considered excitations to the metamaterial, which subsequently vibrates at their corresponding eigenmodes.

\section{FABRICATION \& IMPLEMENTATION}

In this section, we first explain the fabrication process of $U^{2} B$, then elaborate on our overall setup.

\subsection{Mechanical Fabrication}

Fig. 8 shows an exploded view of our transducer design. The transducer consists of a layered piezoceramic structure. We purchased two different types of piezoceramic cylinders from Steminc [4] to build $U^{2} B$. The outer piezoceramic cylinder has a nominal resonance frequency of $17 \mathrm{kHz}$ and has an outer radius of $27 \mathrm{~mm}$, inner radius of $23.5 \mathrm{~mm}$, and height of $40 \mathrm{~mm}$ [48]. The inner piezoceramic cylinder has a nominal resonance frequency of $30 \mathrm{kHz}$ and has an outer radius of $18 \mathrm{~mm}$, inner radius of $15.5 \mathrm{~mm}$, and height of $20 \mathrm{~mm}$ [47]; we stacked two of the inner piezoceramic cylinders and soldered them together to obtain the same height as the outer cylinder. We then soldered wires to the inner and outer surfaces of each of the cylindrical layers, resulting in a total of four terminals for each $U^{2} B$ transducer. To couple the vibration of the two piezoceramic cylinders, we filled the space between them with polyurethane. It is very important to ensure that this polyurethane coupling layer is entirely filled such that it is in contact with the walls of both piezoceramic layers. Otherwise, the possible delamination may significantly deteriorate the coupled vibration mode.

The fabrication process of $U^{2} B$ starts by 3D printing the base and cap of the transducer. Then, we laser cut a polyurethane gasket with $2 \mathrm{~mm}$ thickness to match the size of each of the two cylinders.



(a) Pre-potting Components



(b) Potted Transducer
Figure 9-Fabricated $U^{2} B$ transducer. (a) shows the different components of a $U^{2} B$ transducer prior to potting. (b) shows one of our potted $U^{2} B$ transducers which was tested in the Charles River.

We placed the gasket and then the piezoceramic cylinders in their designated locations on the base, then screwed and tightened the cap and base together. This design process ensures that the inside of the internal piezo remains air-backed even after dipping a $U^{2} B$ node in the water. This design methodology, referred as air-backed design, has been shown to yield to better performance [20].

Next, the capped piezocylinders were placed inside the $3 \mathrm{D}$ printed mold, and we poured the polyurethane WC-575A mixture from BJB enterprise into the cylinder mold [5]. We used a transparent cylinder mold to ensure that the outer surface of the entire structure is covered by the polyurethane mixture, which is needed to insulate it from the surrounding environment. Notice that the transducer base, as shown in Fig. 8, has several openings in the region sandwiched between the two piezoceramic cylinders. These openings guide the poured polyurethane polymer to completely fill the gap and spacing between two piezoceramic layers. Afterwards, the structure is placed for 12 hours inside a pressure chamber at a pressure of 60psi to remove any bubbles from the polyurethane solution. We also experimented with using a vacuum chamber instead of a pressure chamber to remove the bubbles; however, this approach was less effective in removing the bubbles because of the high viscosity of the polyurethane mixture.

Fig. 9 shows the transducer before and after potting. In Fig. 9(a), the inner and outer piezoceramic cylinders are placed on the transducer's base, and are separated by empty space (this separation is eventually filled with the polyurethane mixture for coupling); the figure also shows the outer mold and top cap of the transducer. Fig. 9(b) shows the potted transducer with four electrodes; the electrodes are connected to the inner and outer surfaces of each of the two piezoceramic layers. This enables experimenting with different configurations for backscatter and energy harvesting. The total cost of each fabricated node is around $\$ 120$, which includes the cost of the outer piezo (\$41) and the cost of the two inner piezos ( $\$ 35$ each).

\subsection{Hardware Design}

To communicate via backscatter, underwater nodes short and open two terminals of a piezoelectric transducer as shown in Fig. 2. While a single-layer transducer has only two terminals (positive and negative terminals of the piezoceramic cylinder), $U^{2} B$ 's multi-layer design has four terminals - two for each of its active layers. This provides different options for backscattering by shorting and opening any of the two terminals.

To enable backscatter while at the same time allowing $U^{2} B$ to continuously harvest energy, we determined that the best approach is 
to connect the modulating transistor across the terminals of the outer layer while connecting energy harvesting circuit across the terminals of the inner layers. This approach allows $U^{2} B$ to continuously operate without the need to alternate periods of energy harvesting and backscatter. Aside from the front-end, $U^{2} B$ adopts a similar approach to prior designs in terms of rectification for energy harvesting.

In terms of communication protocols, $U^{2} B$ adopts similar techniques to prior backscatter designs, where the downlink projector encodes bits via PWM and uplink (backscatter) communication is performed via FM0 modulation. In our evaluation, we experimented with both energy-harvesting backscatter and battery-assisted backscatter. Since energy-harvesting is not the focus of this paper, most of our results are reported from battery-assisted backscatter designs. The power consumption for $U^{2} B$ is similar to PAB [20], which ranges from $120 \mu \mathrm{W}$ to $500 \mu \mathrm{W}$. It is important to note that that battery-assisted nature does not discount the importance of backscatter since, being the lowest-power communication technology, it would significantly extend the battery life of underwater communication.

\subsection{Evaluation Setup}

Our evaluation setup is composed of three main components: an acoustic projector, a hydrophone receiver, and a number of $U^{2} B$ nodes. Since we explained the design and fabrication of $U^{2} B$ in previous section, below we will explain the other two main components along with the environment where testing is performed.

(a) Transmitter. To generate an acoustic signal, we used one of our in-house fabricated $U^{2} B$ transducers (discussed in the previous section) as a projector. To drive the node as a projector, a sine wave with the desired amplitude and frequency is first generated using the Siglent SDG1032X arbitrary waveform generator [44]. Next, the output of the signal generator is fed to an XLi2500 Two-channel $750 \mathrm{~W}$ power amplifier [7]. The output of the amplifier is connected to the terminals of the outer piezo in differential mode.

(b) Receiver. To record the signal, we used omnidirectional Reson TC 4014 hydrophone [49] (flat response from $15 \mathrm{~Hz}$ to $480 \mathrm{kHz}$ ) with the sensitivity of $-180 \mathrm{~dB}$ re: $1 \mathrm{~V} / \mu \mathrm{Pa}$ in the differential mode. The output of the hydrophone is connected to USRP N210 [3] and the data is collected with a sampling rate of $1 \mathrm{MSps}$. To capture the differential output of the hydrophone, we used two LFRX daughterboards [2] whose grounds are connected together. Our decoding is performed offline in MATLAB.

(c) Testing Environments. Unless otherwise noted, all of our experiments were performed in a river. In the river, we chose the test location based on a number of factors including accessibility, depth, and water current. Some of our experiments were performed on rainy and snowy days. While changes in weather impacted the SNR and channel coherence time, $U^{2} B$ was capable of operating correctly across different weather conditions.

\section{RESULTS}

To evaluate the performance of $U^{2} B$, we performed controlled and uncontrolled experiments in the river as described in $\$ 4.3$. Our experiments tested $U^{2} B$ 's throughput, communication range, and its

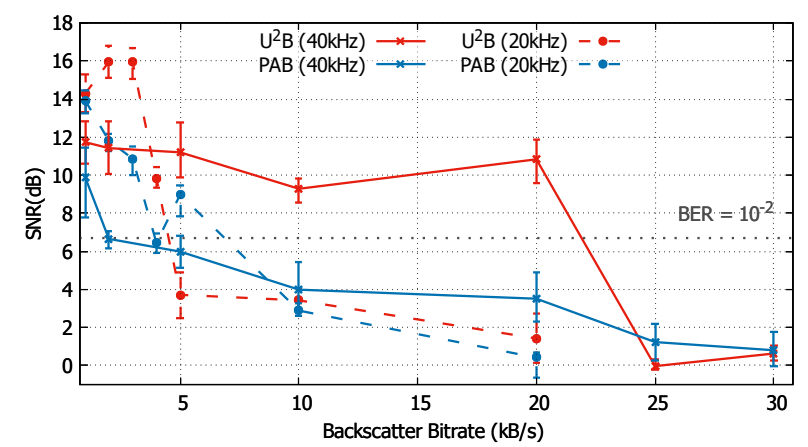

Figure 10 - SNR vs Bitrate. The figure plots the median SNR as a function of bitrate for both $U^{2} B$ (in red) and PAB (in blue) at two different downlink center frequencies: $20 \mathrm{kHz}$ (dashed line) and $40 \mathrm{kHz}$ (solid line). The error bars represents the $25^{\text {th }}$ and $75^{t h}$ percentile and the dotted gray horizontal line represents the SNR needed to get a BER of $10^{-2}$

ability to enable concurrent transmissions. We performed around 400 experimental trials in total. We varied the backscatter rate, location, and depth of $U^{2} B$ 's transducers throughout these experiments.

\subsection{Throughput}

First, we are interested in evaluating $U^{2} B$ 's throughput, and comparing its throughput to the state-of-the-art underwater backscatter system, PAB. To evaluate $U^{2} B$ 's ability to communicate across different bitrates, we fixed the locations of the projector, hydrophone, and backscatter node, and we varied the backscatter bitrate. We repeated the same evaluation for both $U^{2} B$ and PAB. We also tested each of them at two different downlink center frequencies: $20 \mathrm{kHz}$ and $40 \mathrm{kHz}$. In each of our experimental trials, the hydrophone receives the backscatter node's packet and computes the SNR of the received signal. The SNR is computed as the ratio of the signal power (i.e., the square of the channel estimate) to the noise power.

Fig. 10 plots the median SNR as a function of the bitrate for $\mathrm{PAB}$ (in blue) and $U^{2} B$ (in red) at the two center frequencies mentioned earlier. The dotted horizontal line in the figure represents the SNR needed to achieve a throughput of $10^{-2}$, which is considered a standard threshold for reliable underwater communication [54]. We make the following remarks:

- $U^{2} B$ maintains high SNR (i.e., above the dashed lines) at throughputs up to $20 \mathrm{kbps}$. In contrast, the PAB baseline cannot achieve good SNR above $5 \mathrm{kbps}$. These results are in line with those reported in the PAB paper [20] and demonstrate that $U^{2} B$ increases the throughput by about $4-5 \times$ in comparison to the state-of-the-art baseline. This result is expected because $U^{2} B$ 's transducer enjoys a much wider bandwidth as we demonstrated experimentally in Fig. 6.

- The baseline, PAB, performs better when the downlink frequency is at $20 \mathrm{kHz}$ vs at $40 \mathrm{kHz}$. This behavior is also expected according to the characteristic curve in Fig. 6, which showed that the system has a higher SNR around the $20 \mathrm{kHz}$ region, which matches its primary resonance. ${ }^{9}$ In contrast, $U^{2} B$ performs better when the center frequency is $40 \mathrm{kHz}$, a result that is also expected since

\footnotetext{
${ }^{9}$ Note that the SNR from Fig. 6 is that of a single-carrier as described in $\S 3$, which is different from the communication SNR described in this section.
} 


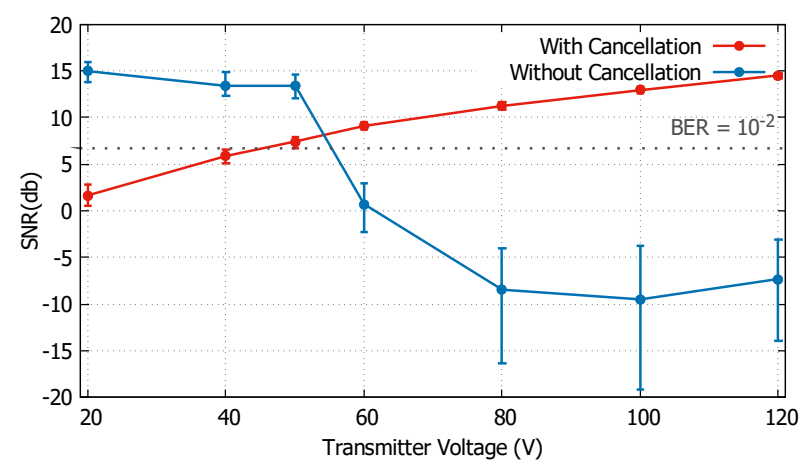

Figure 11-Self-Interference Cancellation. The figure plots the median SNR with (red) and without (blue) self-interference cancellation as a function of transmitter voltage. The error bars represent the $10^{t h}$ and $90^{t h}$ percentile. The dotted line represents the SNR needed to get a BER value of $10^{-2}$.

it has high SNR between $10 \mathrm{kHz}$ and $60 \mathrm{kHz}$ according to its characteristic curve.

- Both $U^{2} B$ and PAB follow similar SNR curves when the downlink frequency is at $20 \mathrm{kHz}$. This is also expected since their characteristic curves are much closer in the bandwidth surrounding $20 \mathrm{kHz}$. Here, $U^{2} B$ achieves slightly higher SNR because it is more efficient even at lower frequencies as described $\S 3.1$.

- Beyond $20 \mathrm{kbps}$, the SNR drops close to $0 \mathrm{~dB}$ for both $U^{2} B$ and PAB. This is expected because the backscatter node becomes inefficient beyond $60 \mathrm{kHz}$ (i.e., $40 \mathrm{kHz}$ center frequency $+20 \mathrm{kHz}$ backscatter rate) which can also be seen from the characteristic curve in Fig. 6.

\subsection{Range}

(a) Self-Interference Cancellation. Recall that $U^{2} B$ employs selfinterference cancellation in order to mitigate the direct path from projector to hydrophone and enable communication with further away nodes. Specifically, by canceling self-interference, the projector can transmit signals at a higher power (without saturating the hydrophone) and receive stronger SNR from the backscatter node.

To assess the benefit of self-interference cancellation, we ran two kinds of experiments: one with and one without the filtering mechanism described in \$3.2. In each kind of experiment, the projector transmits a downlink signal at $40 \mathrm{kHz}$, and the backscatter node is set to shift its response out-of-band by $20 \mathrm{kHz}$. The projector, hydrophone, and backscatter node were all placed within a short distance of about $1 \mathrm{~m}$ in order to better understand the effect of the direct bath and the benefits of self-interference cancellation. In each of the two experiments, we increased the transmitted power (voltage) fed into the power amplifier described in $\S 4.3$, and we measured the SNR of the received backscatter signal.

Fig. 11 plots the SNR as a function of the input voltage to the transducer. We make the following observations:

- Without self-interference cancellation, the SNR starts around $15 \mathrm{~dB}$ but experiences a sharp decline around $50 \mathrm{~V}$ and plateaus around $-7 \mathrm{~dB}$ beyond $80 \mathrm{~V}$. This is because when the input voltage to the transducer exceeds $50 \mathrm{~V}$, the receiver's ADC clips, preventing us from being able to decode the received packet. In such scenarios, the strong self-interference pushes the backscatter signal outside of the dynamic range of the receiver, and the backscatter signal becomes undetectable. As a result, the system can achieve reliable communication (i.e., BER below $10^{-2}$ ) only for low values of transmitter voltages.

- With self-interference cancellation, the SNR starts around $2 \mathrm{~dB}$ and continuously increases with increased transmit voltage, following the theoretically predicted improvement in backscatter SNR. This is because a higher transmit voltage results in a stronger reflection, which in turn leads to high backscatter SNR. This result demonstrates that self-interference cancellation can indeed mitigate the direct path and keep the backscatter response within the dynamic range of the receiver's ADC. This result also demonstrates that $U^{2} B$ 's self-interference cancellation mechanism allows increasing the backscatter SNR and indicates the potential to communicate with further away backscatter nodes.

- Interestingly, at low transmit voltage, the configuration without self-interference cancellation outperforms that with selfinterference cancellation. Specifically, the SNR around $20 \mathrm{~V}$ is around $15 \mathrm{~dB}$ without cancellation but only $2 \mathrm{~dB}$ with cancellation. This is because the low-pass filter adds an insertion loss which degrades the receive SNR. Note that it is straightforward to overcome this loss by adding an LNA at the receiver (or by amplifying the transmit signal).

- One might wonder whether self-interference cancellation is indeed useful if the highest SNR with amplification (i.e., 120V) is similar to that with no amplification (i.e., 20V) in the absence of cancellation. Note, however, that this is due to an reduction in SNR arising from the filter's insertion loss. By accounting for this loss and adding an LNA, the received signal with cancellation would be around $13 \mathrm{~dB}$ higher than the highest backscatter SNR without cancellation and results in increasing the communication range as we demonstrate below.

(b) Communication Range. Next, we would like to evaluate the range at which we can reliably communicate with $U^{2} B$. In this experiment, we varied the distance between the backscatter node and the hydrophone/projector pair. The hydrophone and projector were co-located (1m apart) and the backscatter device was moved further away (up to $62 \mathrm{~m}$ round trip). At each distance, we applied a CDMA-style code and measured the received signal's SNR after coding. To deal with the low power of the backscatter response, we applied codes of different lengths: 30s, 60s, and 120s. We repeated each experiment at least 3 times at each rate and distance.

Fig. 12 plots the SNR as a function of distance for each of the coding rates. We make the following remarks:

- The figure shows that $U^{2} B$ nodes can be used to communicate up to $60 \mathrm{~m}(\mathrm{SNR}>3 \mathrm{~dB}) .{ }^{10}$ Such range represents more than $6 \times$ improvement over the baseline (PAB) [20].

- The SNR decreases with distance across different coding rates. This is expected because the signal power decays with distance. Moreover, the trend follows the theoretically expected decay resulting from the round-trip path loss.

\footnotetext{
${ }^{10}$ Note that we used the $3 \mathrm{~dB}$ threshold here instead of the $7 \mathrm{~dB}$ one since we use coding.
} 




Figure 12-Backscatter SNR vs Distance. The figure plots the backscatter SNR as a function of distance for different coding rates. Error bars indicate standard deviation, and solid lines indicate smoothed curves.

- Applying longer codes enables us to communicate over further distances. Specifically, 30s, 60s, and 120s codes can communicate up to $32 \mathrm{~m}, 48 \mathrm{~m}$ and $60 \mathrm{~m}$ respectively. Moreover, since our USRP receiver is limited to a 14-bit ADC, it would be possible to communicate over longer ranges with higher-bit ADCs due to the higher dynamic range.

\subsection{Scaling Performance}

Finally, we would like to evaluate $U^{2} B$ 's ability to scale to a large number of nodes and enable concurrent decoding. Recall from $\$ 3.3$ that $U^{2} B$ employs an FDMA-based mechanism that allows it to allocate different frequency channels to different backscatter nodes and decode each independently.

To evaluate this capability, we ran a controlled experiment in an indoor pool whose dimensions are $3 \mathrm{~m} \times 4 \mathrm{~m} \times 1.5 \mathrm{~m}$. In each experimental trial, we varied the number of backscattering nodes from 1 to 10. Each node was assigned a different frequency channel as per the protocol described in $\$ 3.3$. The hydrophone receives the concurrent responses, applies appropriate software-based filters to isolate them, and decodes each of them separately. We repeated each experimental trial at least 10 times for each number of nodes. For any given number of nodes, we varied the location of the nodes across different trials to average out the effect of the frequency selective channel.

Fig. 13 plots the median BER and SNR as a function of the number of nodes. We make the following observations:

- The SNR starts at around $18 \mathrm{~dB}$ for one node and gradually drops as we add more $U^{2} B$ nodes to the environment. This gradual degradation is due to the residual interference caused by neighboring nodes. Nonetheless, even with 10 nodes, $U^{2} B$ can achieve a median BER of $10^{-1}$. This result shows that $U^{2} B$ can indeed scale up to 10 concurrent nodes despite their close proximity (and interference), thus demonstrating a $5 \times$ improvement over the state-of-the-art baseline (PAB) [20].

- It is also interesting to note here that the BER doesn't change much as we add more nodes. This is because we operate these nodes at a relatively low bitrate of $100 \mathrm{kbps}$.

- Finally, it is worth noting that the above performance is achieve even though all the nodes are in close proximity. It is possible to

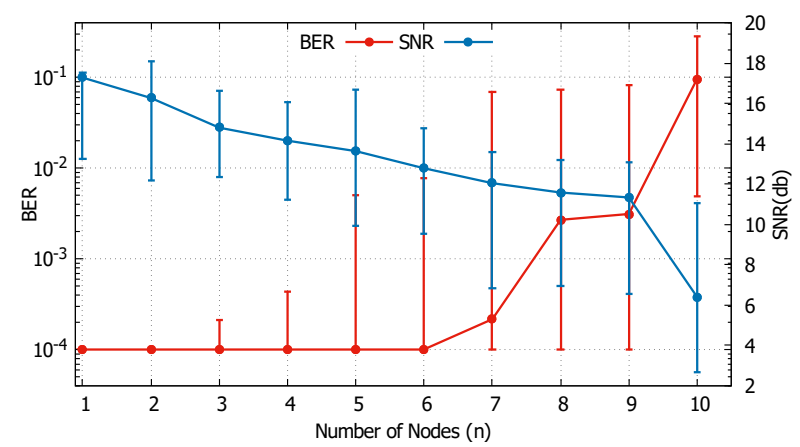

Figure 13-BER and SNR vs Concurrent Transmissions. The figure plots the median BER and SNR as a function of the number of concurrent transmissions. The error bars represent the $25^{\text {th }}$ and $75^{\text {th }}$ percentile.

scale the system to more concurrent transmissions by exploiting spatial reuse.

\section{RELATED WORK}

(a) Low-power underwater networking. Recent research on lowpower underwater networking has resulted in various proposals for energy harvesting, duty cycling, and low-power communication [15, 34]. Among these proposals, underwater backscatter has emerged as the most energy-efficient communication primitive by demonstrating orders of magnitude reduction in energy consumption in comparison to other underwater communication technologies [20]. However, prior work on underwater backscatter relied on piezoelectric resonators, which limited its bandwidth, throughput, range, and scale of operation. $U^{2} B$ directly builds on this work and introduces UWB to the underwater backscatter problem. This results in achieving $5 \mathrm{x}$ more throughput, range, and scale than the sate-of-the-art system as we showed empirically in $\$ 5$.

(b) Transducer Design. Underwater transducer design is a wellstudied area of research that dates back to the development of submarines in WWI. There are many forms of underwater transducers including multi-layer, micro-fabricated, and composite designs $[6,9,35,37,39,46] . U^{2} B$ differs from prior acoustic transducers in both design and functionality. In terms of design, the two cylindrical active layers with a polymer layer between them is, to the best of our knowledge, unique and novel. In terms of functionality, $U^{2} B$ maximizes the backscatter SNR (over a wide bandwidth) - i.e., the difference between two acoustic impedance states; in contrast, classical underwater transducer designs focus on transmitting and/or receiving, and thus they need to only maximize the direct SNR - i.e., a single acoustic impedance.

(c) Acoustic metamaterials is a relatively new field that has introduced novel mathematical frameworks to understand and design acoustic devices and has also enabled interesting new applications such as sound silencing [12], acoustic beam forming [13, 26], and broadband impedance matching [25]. $U^{2} B$ uses these frameworks in order to optimize for the parameter described above: namely the backscatter SNR over a wide bandwidth. Specifically, in $\$ 2.2, \S 3.1$ and $\S \mathrm{A}$, we show that it is possible to optimize the difference between acoustic impedances (hence the backscatter SNR) over wide 
bandwidth by synthesizing various resonances. To the best of our knowledge, this is the first application of metamaterials to acoustic backscatter communication.

(d) RF Systems: $U^{2} B$ is related to two areas of research in RF systems: backscatter networking and UWB communication. The past few years have seen significant progress in RF backscatter networking, including high throughput, full-duplex, and FDMA designs $[17,27,52,56]$. Such systems, however, are not suitable for underwater environments because radio signals die exponentially in water, and bringing their benefits to underwater environments requires addressing new constraints that are imposed by this medium and the acoustic nature of communications. Similarly, UWB systems have been well-studied for RF communication, but are also unsuitable for underwater environments for the same reasons. $U^{2} B$ 's contributions are orthogonal to past work on RF and aim at bringing the benefits of state-of-the-art RF backscatter designs and UWB communication to the subsea IoT domain.

(e) High-Throughput Ocean Communication Systems: Past work has considered increasing the throughput of ocean communication systems by using optical communication $[33,55]$ or highfrequency ultrasound (in the MHz range) [9, 29]. Such systems are too power-hungry for the subsea IoT domain, and they suffer from a limited communication range and directionality due to their higher frequencies of operation. $U^{2} B$ targets a different set of application domain than these past systems. Finally, it is worth noting that some research has looked into a theoretical understanding of UWB acoustic communication [51]; such work is orthogonal to $U^{2} B$ 's contributions and provides channel modeling rather than proposing systems that can enable ultrawideband underwater backscatter.

\section{CONCLUSION}

Motivated by recent advances in underwater backscatter and ultralow power networking, this paper introduces a new design, $U^{2} B$, that marks a significant step forward in this domain. The design bridges recent advances in metamaterials to the underwater backscatter problem, and demonstrates significant improvements over state-of-the-art proposals. As the research evolves, we hope that these techniques would enable truly ubiquitous subsea IoT systems that can be used for climate change monitoring, marine life sensing, and ocean exploration.

Acknowledgments. We thank Prof. Michael Triantafyllou and Dr. Thomas Consi at the MIT Sea Grant and Dr. Mike Benjamin at the MIT Sailing Pavilion for their support. We also thank Tara Boroushaki, the Signal Kinetics group, and the anonymous SIGCOMM reviewers and shepherd for their helpful feedback on the manuscript. This research is supported by the Office of Naval Research, the MIT Media Lab, and the Doherty Chair in Ocean Utilization.

This work does not raise any ethical issues.

\section{REFERENCES}

[1] COMSOL Multiphysics. https://www.comsol.com/.

[2] LFRX daughterboard. http://www.ettus.com. ettus inc.

[3] usrp n210. http://www.ettus.com. ettus inc.

[4] Steminc. https://www.steminc.com/, 2020.
[5] 2015., B. E. Water clear shore 70 a polyurethane elastomer. https:// bjbenterprises.com/index.php/wc-575-a-b/, 2015.

[6] Butler, J., AND C.H., S. Transducers and Arrays for Underwater Sound. Springer, 2016

[7] CROWN. Crown Audio Power Amplifier. Part No. XLi-2500 750W https: //www.crownaudio.com/en/products/xli-2500.

[8] DARPA. Ocean of Things Aims to Expand Maritime Awareness across Open Seas. https://www.darpa.mil/news-events/2017-12-06, 2017.

[9] Demirors, E., Shi, J., Duong, A., Dave, N., Guida, R., Herrera, B., Pop, F., Chen, G., Casella, C., TAdayon, S., ET Al. The seanet project: Toward a programmable internet of underwater things. In 2018 Fourth Underwater Communications and Networking Conference (UComms) (2018), IEEE, pp. 1-5.

[10] Dong, H., Wang, H., Shen, X., Huang, Z., And Ma, S. Detection of underwater weak signal via matched stochastic resonance. In OCEANS 2017 Aberdeen (June 2017), pp. 1-7.

[11] Fontana, R., Ameti, A., Richley, E., Beard, L., And Guy, D. Recent advances in ultra wideband communications systems. In 2002 IEEE Conference on Ultra Wideband Systems and Technologies (IEEE Cat. No.02EX580) (May 2002), pp. 129-133.

[12] Ghaffarivardavagh, R., Nikolajczyk, J., Anderson, S., And Zhang, $\mathrm{X}$. Ultra-open acoustic metamaterial silencer based on fano-like interference. Phys. Rev. B 99 (Jan 2019), 024302.

[13] Ghaffarivardavagh, R., Nikolajczyk, J., Glynn Holt, R., Anderson, S., AND ZHANG, X. Horn-like space-coiling metamaterials toward simultaneous phase and amplitude modulation. Nature Communications 9, 1 (2018).

[14] Google. Oil and Gas Equipment Monitoring and Analytics. https: //cloud.google.com/solutions/oil-and-gas-equipment-monitoring-and-analytics, 2019.

[15] Guida, R., Demirors, E., Dave, N., Rodowicz, J., and Melodia, T. An acoustically powered battery-less internet of underwater things platform. In 2018 Fourth Underwater Communications and Networking Conference (UComms) (2018), IEEE, pp. 1-5.

[16] Heidemann, J., Wei Ye, Wills, J., Syed, A., and Yuan Li. Research challenges and applications for underwater sensor networking. In IEEE Wireless Communications and Networking Conference, 2006. WCNC 2006. (April 2006), vol. 1 , pp. 228-235.

[17] Hu, P., ZhANG, P., AND GANESAN, D. Laissez-faire: Fully asymmetric backscatter communication. ACM SIGCOMM Computer Communication Review 45, 4 (2015), 255-267.

[18] Huang, X., Lowe, D., Gandia, R., And Dutkiewicz, E. An impulse ultra-wideband system capable of concurrent transmission and reception, part i: Requirements and innovations. In 2006 International Conference on Communications, Circuits and Systems (June 2006), vol. 2, pp. 1251-1255.

[19] Huang, X., Lowe, D., Gandia, R., And DutKiewicz, E. An impulse ultrawideband system capable of concurrent transmission and reception, part ii: Design and performance. In 2006 International Conference on Communications, Circuits and Systems (June 2006), vol. 2, pp. 1256-1260.

[20] JANG, J., AND ADIB, F. Underwater backscatter networking. In Proceedings of the ACM Special Interest Group on Data Communication (2019), ACM, pp. 187199.

[21] Jepsen, N., Thorstad, E. B., Havn, T., and Lucas, M. C. The use of external electronic tags on fish: an evaluation of tag retention and tagging effects. Animal Biotelemetry 3, 1 (2015), 49.

[22] KaO, C.-C., LIN, Y.-S., Wu, G.-D., AND HuANG, C.-J. A comprehensive study on the internet of underwater things: applications, challenges, and channel models. Sensors 17, 7 (2017), 1477.

[23] Li, H., Tian, C., Lu, J., Myjak, M., Martinez, J., Brown, R., And Z.D., D. An energy harvesting underwater acoustic transmitter for aquatic animals. Scientific Reports 6, 33804 (2016)

[24] Liou, E.-C., KaO, C.-C., Chang, C.-H., Lin, Y.-S., And Huang, C.-J. Internet of underwater things: Challenges and routing protocols. In 2018 IEEE International Conference on Applied System Invention (ICASI) (2018), IEEE, pp. 1171-1174.

[25] LiU, C., LUO, J., AND LAI, Y. Acoustic metamaterials with broadband and wide-angle impedance matching. Phys. Rev. Materials 2 (Apr 2018), 045201.

[26] MA, G., AND SHENG, P. Acoustic metamaterials: From local resonances to broad horizons. Science Advances 2, 2 (2016).

[27] Ma, Y., Luo, Z., Steiger, C., Traverso, G., And Adib, F. Enabling deeptissue networking for miniature medical devices. In Proceedings of the 2018 Conference of the ACM Special Interest Group on Data Communication (2018), ACM, pp. 417-431.

[28] MA, Y., SElBy, N., AND AdiB, F. Drone relays for battery-free networks. In ACM SIGCOMM (2017).

[29] Martins, M., Correia, V., Lanceros-Mendez, S., Cabral, J., And RochA, J. Comparative finite element analyses of piezoelectric ceramics and polymers at high frequency for underwater wireless communications. Procedia Engineering 5 (2010), 99-102. 
[30] MBARI. Wave Glider Hot Spot Enabling autonomous devices to talk back. https://www.mbari.org/technology/emerging-current-tools/communications/ wave-glider-based-communications-hotspot/, 2019.

[31] MÜLLER, P. C., AND GÜRGÖZE, M. Natural frequencies of a multi-degree-offreedom vibration system. In PAMM: Proceedings in Applied Mathematics and Mechanics (2006), vol. 6, Wiley Online Library, pp. 319-320.

[32] OHI. Ocean Health Index. http://www.oceanhealthindex.org/, 2020.

[33] Oubei, H. M., Duran, J. R., Janjua, B., Wang, H.-Y., Tsai, C.-T., Chi, Y.-C., NG, T. K., KuO, H.-C., HE, J.-H., Alouini, M.-S., ET AL. 4.8 gbit/s 16 qam-ofdm transmission based on compact 450-nm laser for underwater wireless optical communication. Optics express 23, 18 (2015), 23302-23309.

[34] Pessoa, L. M., Pereira, M. R., Santos, H. M., and Salgado, H. M. Simulation and experimental evaluation of a resonant magnetic wireless power transfer system for seawater operation. In OCEANS 2016 - Shanghai (April 2016), pp. 1-5.

[35] Qiu, Y., Gigliotti, J. V., Wallace, M., Griggio, F., Demore, C. E., Cochran, S., AND Trolier-MCKInSTRY, S. Piezoelectric micromachined ultrasound transducer (pmut) arrays for integrated sensing, actuation and imaging. Sensors 15, 4 (2015), 8020-8041.

[36] RAJESWARI JAYARAMAN. Beyond IoT: Internet of Underwater Things to Network the Oceans. https://www.prescouter.com/2017/06/internet-of-underwater-things/, 2017.

[37] Ramesh, R., Prasad, C. D., Kumar, T. V., GaVane, L., and VishnubHATLA, R. Experimental and finite element modelling studies on single-layer and multi-layer 1-3 piezocomposite transducers. Ultrasonics 44, 4 (2006), 341-349.

[38] Romme, J., AND KULl, B. On the relation between bandwidth and robustnes of indoor uwb communication. In IEEE Conference on Ultra Wideband Systems and Technologies, 2003 (Nov 2003), pp. 255-259.

[39] Sadeghpour, S., Pobedinskas, P., Haenen, K., and Puers, R. A piezoelectric micromachined ultrasound transducers (pmut) array, for wide bandwidth underwater communication applications. In Multidisciplinary Digital Publishing Institute Proceedings (2017), vol. 1, p. 364.

[40] SCHROCK. Fact or fiction?: An opera singer's piercing voice can shatte glass. https://www.scientificamerican.com/article/fact-or-fiction-opera-singercan-shatter-glass/.

[41] SCIENCE, H. How to break glass with your voice. https://www.youtube.com/ watch? $\mathrm{v}=\mathrm{rRZT} 7 \mathrm{xO} 5 \mathrm{KN} 4$

[42] SEIFERT, J. Ocean vital signs are stable, but bill of health isn't clean. NCEAS. https://www.nceas.ucsb.edu/news/ocean-vital-signs-are-stableno-clean-bill-health, 2017.

[43] ShKel, A. A., Baumgartel, L., AND Kim, E. S. A resonant piezoelectric microphone array for detection of acoustic signatures in noisy environments. In 2015 28th IEEE International Conference on Micro Electro Mechanical Systems (MEMS) (Jan 2015), pp. 917-920.

[44] SIGLENT. Siglent Arbitrary Waveform Generators. Part No. SDG1032X https: //siglentna.com/product/sdg1032x/.

[45] SIWIAK, K. Ultra-wide band radio: introducing a new technology. In IEEE VTS 53rd Vehicular Technology Conference, Spring 2001. Proceedings (Cat. No.01CH37202) (May 2001), vol. 2, pp. 1088-1093 vol.2.

[46] SMIth, W. A. Modeling 1-3 composite piezoelectrics: hydrostatic response. IEEE transactions on ultrasonics, ferroelectrics, and frequency control 40, 1 (1993), $41-49$.

[47] Steminc. Piezo ceramic cylinder 36x31x20mm 30 khz. (2020). Part No SMC3631T20111. https://www.steminc.com/PZT/en/piezo-ceramic-cylinder36x31x20mm-30-khz, 2020.

[48] Steminc. Piezo ceramic cylinder 54.1x47x40mm 17 khz. (2020). Part No SMC5447T40111. https://www.steminc.com/PZT/en/piezo-ceramic-cylinder$541 \times 47 \times 40 \mathrm{~mm}-17-\mathrm{khz}, 2020$.

[49] TELEDYNEMARINE. Reson TC-4014 Hydrophone. Part No. TC-4014 http: //www.teledynemarine.com/reson-tc-4014.

[50] TONOLINI, F., AND ADIB, F. Networking across boundaries: enabling wireless communication through the water-air interface. In Proceedings of the 2018 Conference of the ACM Special Interest Group on Data Communication (2018), ACM, pp. 117-131.

[51] VAN WALReE, P. A., AND OtNes, R. Ultrawideband underwater acoustic communication channels. IEEE Journal of Oceanic Engineering 38, 4 (2013), 678-688.

[52] Vasisht, D., Zhang, G., Abari, O., Lu, H.-M., Flanz, J., And Katabi, D. In-body backscatter communication and localization. In Proceedings of the 2018 Conference of the ACM Special Interest Group on Data Communication (2018), ACM, pp. 132-146.

[53] WHOI. WHOI Micro-modem. https://acomms.whoi.edu/micro-modem/.

[54] Woodward, B., AND SARI, H. Digital underwater acoustic voice communications. IEEE Journal of Oceanic Engineering 21, 2 (1996), 181-192.

[55] Yu FAi Fung, MingJun DaI, AND ERCAN, M. F. Underwater short range free space optical communication for a robotic swarm. In 2009 4th International
Conference on Autonomous Robots and Agents (Feb 2009), pp. 529-532.

[56] Zhang, P., Rostami, M., Hu, P., and Ganesan, D. Enabling practica backscatter communication for on-body sensors. In Proceedings of the 2016 conference on ACM SIGCOMM 2016 Conference (2016), ACM, pp. 370-383.

[57] ZHU, X. Monitoring environmental conditions near underwater datacenters using Deep Learning. Microsoft, 2018.

\section{A APPENDIX}

Appendices are supporting material that has not been peer-reviewed

The interaction of an incoming acoustic wave with the piezoelectric transducer is determined by how the transducer wall vibrates under the wave. To model this interaction, we present a simplified solution assuming the boundaries of piezoelectric cylinder are moving only in one degree of freedom. By analogy to the spring-mass systems, the equation of motion of the Piezoceramic transducer under external time harmonic excitation may be written as:

$$
i \omega m U+r U+k U / i \omega=S\left(P_{i n}+P_{r e f}-P_{t r}\right)
$$

where $m, r$ and $k$ are the equivalent mass, resistance and stiffness of the Piezo-transducer's lumped model, respectively; $U$ and $S$ are the velocity of the transducer wall and the area of the transducer's surface, respectively; $P_{i n}, P_{r e f}$, and $P_{t r}$ denote the incident acoustic pressure, reflected acoustic pressure and transmitted acoustic pressure, respectively; and, $\omega$ is the angular frequency. By defining the parameters for the unity transducer's surface area, one may rewrite the Eq. 3 as:

$$
[i \omega M+R+K / i \omega] U=P_{\text {in }}+P_{\text {ref }}-P_{t r}
$$

where $M=m / S, R=r / S$, and $K=k / S$.

Applying the continuity of velocity on the transducer's surface, we can write:

$$
U=U_{\text {in }}+U_{\text {ref }}=U_{t r}
$$

Assuming plane wave propagation, we can write:

$$
\begin{gathered}
U_{\text {in }}=\frac{P_{\text {in }}}{Z_{\text {water }}} \\
U_{\text {ref }}=-\frac{P_{\text {ref }}}{Z_{\text {water }}} \\
U_{\text {tr }}=\frac{P_{\text {tr }}}{Z_{\text {air }}} \\
U=\frac{P_{\text {total }}}{Z}=\frac{P_{\text {in }}+P_{r e f}-P_{t r}}{Z}
\end{gathered}
$$

where $Z_{\text {air }}, Z_{\text {water }}$, and $Z$ are the acoustic impedances of air, water, and the piezoelectric respectively. As per Eq. 3 and Eq. 9, we can derive the effective acoustic impedance of the piezoelectric as:

$$
Z=i \omega M+R+K / i \omega
$$

As per Eqs. 4-10, we obtain the following relationships between incident and reflected pressure:

$$
\begin{gathered}
\left(Z+Z_{\text {air }}\right) U=P_{\text {in }}+P_{\text {ref }} \\
\left(Z_{\text {water }}\right) U=P_{\text {in }}-P_{\text {ref }}
\end{gathered}
$$

Thus, the acoustic reflection coefficient of an air-backed underwater piezoelectric transducer can be written as:

$$
\text { Reflection coefficient }=\frac{P_{\text {ref }}}{P_{\text {in }}}=\frac{Z+Z_{\text {air }}-Z_{\text {water }}}{Z+Z_{\text {air }}+Z_{\text {water }}}
$$

